$1 \quad$ Risk Factors for Klebsiella infections among hospitalized patients with Pre-Existing

\title{
2 Colonization
}

$4 \quad$ Running Title: Risk factors for Klebsiella infections

6 Krishna Rao ${ }^{1}$, Alieysa Patel ${ }^{2}$, Yuang Sun $^{2}$, Jay Vornhagen ${ }^{2}$, Jonathan Motyka ${ }^{1}$, Abigail

7 Collingwood ${ }^{2}$, Alexandra Teodorescu ${ }^{2}$, Lili Zhao ${ }^{3}$, Keith S. Kaye ${ }^{1}$, Michael Bachman ${ }^{2,4 \#}$

$9{ }^{1}$ Infectious Diseases Division, Department of Internal Medicine, Michigan Medicine; ${ }^{2}$

10 Department of Pathology, Michigan Medicine; ${ }^{3}$ Department of Biostatistics, School of Public

11 Health; ${ }^{4}$ Department of Microbiology and Immunology, Michigan Medicine, Ann Arbor, MI

13 \# Corresponding Author:

14 7510E MSRB 1

151150 W Medical Center Drive

16 Ann Arbor, MI 48109 


\section{Abstract}

19 Background: Klebsiella commonly colonizes the intestinal tract of hospitalized

20 patients and is a leading cause of healthcare-associated infections. Colonization is

21 associated with subsequent infection, but the factors determining this progression are unclear.

22 Methods: Intensive care and hematology/oncology patients were screened for Klebsiella

23 colonization by rectal swab culture and monitored for infection for 90 days after a positive swab.

24 Electronic medical records were analyzed for patient factors associated with subsequent

25 infection, and variables of potential significance in bivariable analysis were used to build a final

26 multivariable model. Concordance between colonizing and infecting isolates was assessed by wzi

27 capsular gene sequencing.

28 Results: Among 2087 hospital encounters from 1978 colonized patients, 90 cases of infection

$29(4.3 \%)$ were identified. Mean time to infection was $20.6 \pm 24.69$ (range 0-91, median 11.5) days.

30 Of 86 typed cases, 68 unique wzi types were identified and 69 cases $(80.2 \%)$ were colonized with

31 an isolate of the same type prior to infection. Based on multivariable modeling, overall

32 comorbidities, depression and low albumin level at the time of rectal swab were independently

33 associated with subsequent Klebsiella infection.

34 Conclusions: Despite the high diversity of colonizing strains of Klebsiella, there is high

35 concordance with subsequent infecting isolates and progression to infection is relatively quick.

36 Readily accessible data from the medical record could be used by clinicians to identify colonized

37 patients at increased risk of subsequent Klebsiella infection. 


\section{Importance}

39 Klebsiella is a leading cause of healthcare-associated infections. Patients who are intestinally

40 colonized with Klebsiella are at significantly increased risk of subsequent infection, but only a

41 subset of colonized patients progress to disease. Colonization offers a potential window of

42 opportunity to intervene and prevent these infections, if the patients at greatest risk could be

43 identified. To identify patient factors associated with infection in colonized patients, we studied

441978 colonized patients. We found that patients with a higher burden of underlying disease in

45 general, depression in particular, and low albumin in a blood test were more likely to be a case of

46 infection. However, these variables did not completely predict infection, suggesting that other

47 host and microbial factors may also be important. The average time to infection was 3 weeks,

48 suggesting that there is time to intervene and prevent Klebsiella infections in hospitalized

49 patients. 


\section{Introduction}

52 Klebsiella pneumoniae is a leading cause of healthcare-associated infections, commonly causing

53 pneumonia, bacteremia, and urinary tract infections (1). With advancements in genomics, it is

54 clear that infections attributed to K. pneumoniae may be caused by related members of a multi-

55 species complex (henceforth Klebsiella). Clinical laboratories can now identify K. variicola,

56 which may have clinical characteristics distinct from K. pneumoniae, but not other complex

57 members such as K. quasipneumoniae $(2,3)$. Klebsiella is also a common colonizer of the

58 gastrointestinal tract (4), creating a reservoir of potential disease-causing bacteria. In fact, among

59 colonized patients who develop Klebsiella infections, the majority of isolates match those found

60 in the gastrointestinal tract $(5,6)$. This concordance between colonizing and invasive isolate can

61 be measured by sequencing of the wzi capsular gene with the same discriminatory power as 7-

62 gene multi-locus sequence typing (6). This tight link between colonization and infection suggests

63 an opportunity for prevention of infection in hospitalized patients, where detection of

64 colonization could trigger an intervention.

66 Despite the strong link between colonization and infection, the risk factors for infection in

67 colonized patients are unclear. A combination of patient and bacterial variables likely determines

68 if a patient will develop a Klebsiella infection. At a population level without assessment of

69 colonization, increased age, male sex, dialysis, chronic liver disease, solid organ transplant, and

70 cancer were associated with Klebsiella bacteremia (7). Klebsiella colonization itself is

71 associated with advanced age as well as vancomycin-resistant enterococci (VRE) colonization

72 (8). We previously found certain Klebsiella genes and patient factors associated with Klebsiella 
73 infection compared to asymptomatic colonization (9). However, this study was limited by a

74 small sample size and unknown colonization status in some infected patients.

76 The goal of this study was to identify risk factors for Klebsiella infection among colonized

77 patients at the time of detectable colonization by a rectal swab. In a cohort study of over 2000

78 hospital encounters with colonized patients, the bacterial species and concordance with

79 subsequent infecting strains was determined. Patient variables were assessed in bivariable

80 analysis and used to create an explanatory model of Klebsiella infection. Our findings indicate

81 that readily accessible chart variables can be used to stratify risk of Klebsiella infection in

82 colonized patients. 


\section{Results}

$84 \quad$ Swab collection and Klebsiella colonization

85 From 5/1/2017 to 12/13/2018, rectal swabs collected for VRE screening were cultured for

86 Klebsiella, identifying 2098 positive swabs from 1978 patients (recruitment goal=2000).

87 Eliminating duplicate swabs from the same patient within 90 days of the enrollment swab, the

88 cutoff for a subsequent infection to meet our case definition,, resulted in a final cohort consisting

89 of 2087 inpatient admissions with baseline Klebsiella colonization among 1978 patients. There

90 was a noticeable seasonal variation in rates of colonization (Figure 1) that appeared to co-vary

91 with the mean daily temperature with increased colonization rates occurring in warmer months.

92 Up to 3 isolates were archived from each colonized patient, yielding 5463 Klebsiella isolates. Of

932087 patient encounters, 1712 admissions (82.03\%) were colonized with K. pneumoniae only,

$94308(14.76 \%)$ were colonized with $K$. variicola only, and 67 (3.21\%) admissions were colonized

95 with both $K$. pneumoniae and $K$. variicola.

$97 \quad$ Clinical cultures and case determinations

98 There were 402 clinical cultures positive for Klebsiella from 262 patients, of which $386(95.8 \%)$

99 were K. pneumoniae and $16(3.97 \%)$ were $K$. variicola. These cultures were assessed for cases of

100 Klebsiella infection based on chart review. 52 blood cultures were reviewed, and $51(98.1 \%)$

101 were considered unique cases of infection (one subject had two positive blood cultures and only

102 the first was counted), of which 37 met the 90-day cutoff and were not duplicates (Table 1). 199

103 urine cultures were reviewed and $46(23.1 \%)$ were considered cases of infection, 22 of which

104 met the 90-day cutoff and were not duplicates. 72 respiratory cultures were reviewed and 40

105 (55.6\%) were considered cases of infection, of which 27 met the 90-day cutoff and were not 
107 cases of infection, of which 4 met the 90-day cutoff and were not duplicates (1 abscess, 2 bile,

108 and 1 tissue). In total, there were 90 infections meeting case definitions for a unique episode

109 within 90 days of the enrollment swab (1 infection occurred 91 days from the index swab but

110 was still included in the analysis), This represents a rate of infection of $4.3 \%$ out of 2087

111 hospital encounters from 1978 Klebsiella colonized patients. Bloodstream infections were most

112 frequent $(37,41.1 \%)$ followed by respiratory $(27,30.0 \%)$, urine $(22,24.4 \%)$ and other sites $(4$,

$1134.4 \%)$.

115 Most infections were caused by K. pneumoniae (86.7\%). However, there was high strain

116 diversity with 68 unique wzi types observed across 90 infections. Despite this diversity, the

117 majority of these infections were concordant with a Klebsiella isolate from the enrollment swab

118 (69/86, 80.2\%, 4 cases had missing wzi data). Urinary tract infections (UTI) had the highest rate

119 of concordance $(86.3 \%)$, bacteremia had a similar rate $(81.1 \%)$ and pneumonia was lowest (63\%;

$120 P=0.11$ ) The mean time from swab to infection was $20.6 \pm 24.69$ (median 11.5, range 0-91) for

121 all infections and 17.7 days \pm 21.98 (median 10, range 0-90) for concordant cases (Table 1). The

122 time to concordant infection (Figure 2) differed by site of infection overall $(P=.001)$ and was

123 significantly longer for UTI $(32.8 \pm 27.56)$ than for bacteremia $(12.0 \pm 17.88 ; P<0.001)$ or

124 pneumonia $(13.4 \pm 14.93 ; P=0.006)$.

126 Among all cases of infection, 19 (21.1\%), 13 were found to be extended spectrum beta-lactamase 127 positive (7 bloodstream infections, 4 respiratory, and 2 UTI), and all 13 infecting strains were $K$.

128 pneumoniae. In addition, 6 cases were carbapenem resistant, including 3 bloodstream and 3 
129 respiratory infections. 5 of these strains were K. pneumoniae and the other was K. variicola .

130 Overall, 17 of these antibiotic-resistant cases were concordant with a colonizing rectal swab.

132 Clinical variables and invasive infection with Klebsiella

133 The initial unadjusted analyses compared cases to controls and numerous variables showed

134 statistically significant differences (Table 2). There were no significant differences in age, sex,

135 or race between cases and controls. The season that Klebsiella colonization was detected on the

136 baseline rectal swab did not associate with increased risk of infection $(P=0.358)$. Comorbid

137 illness, as assessed by a Weighted Elixhauser score, and high-risk antibiotic exposure were both

138 enriched in cases compared to controls (Table 2). Notably, depression has a neutral weight for

139 predicting in-hospital mortality in the Elixhauser score, but it was significantly associated with

140 Klebsiella infection and thus considered separately for the multivariable modeling described

141 below. Invasive devices including urinary catheters and feeding tubes actually had an inverse

142 association with infection and ventilator at baseline trended towards an inverse association

$143(P=0.1)$. A number of medications, including diuretics, antidepressants, Vitamin $\mathrm{D}$,

144 pressors/inotropes, were also associated with Klebsiella infection.

146 Multivariable Model of Klebsiella infection

147 The purposeful selection process resulted in the explanatory model for Klebsiella infection

148 shown in Table 3. Here the baseline features of weighted Elixhauser score, depression, and

149 serum albumin $<2.5 \mathrm{~g} / \mathrm{dL}$ associated with an increased risk of subsequent Klebsiella infection,

150 and use of pressors/inotropes showed a borderline association with increased risk. Prior diuretic,

151 vitamin $\mathrm{D}$, and high-risk antibiotic use did not independently associate with the outcome, but 
152 were retained in the model as confounders. Although depression was selected, anti-depressants

153 were not. The overall fit of the model by area under the receiver operator characteristic curve

154 (AuROC) was 0.74 . The mean predicted risk of infection in cases was $8.61 \%$ vs. $4.35 \%$ in

155 controls, for a discrimination slope of $4.26 \%$ (95\% confidence interval [CI] 2.59\%-5.94\%).

157 For the 69 cases of infection that were concordant by wzi type with the colonizing isolate, we

158 built a separate multivariable model, shifting the cases without concordance to the control group

159 (Table 4). Here the weighted Elixhauser score, depression, and serum albumin $<2.5 \mathrm{~g} / \mathrm{dL}$ were

160 again associated with an increased risk of infection, while baseline serum hemoglobin showed a

161 borderline association such that reduced hemoglobin increased risk. Other potential confounders

162 were not retained. The model AuROC was 0.73 , and the mean predicted risk of infection in cases

163 was $5.6 \%$ vs. $3.44 \%$ in controls for a discrimination slope of $2.16 \%$ (95\% CI $1.31 \%-3 \%$ ). We

164 also conducted a subset analysis by species, and on unadjusted analysis, infection with $K$.

165 pneumoniae vs. K. variicola was associated with an increased risk of infection that did not reach

166 statistical significance (OR 1.81 [0.92, 4.1], $P=.114)$. 


\section{Discussion}

168 This study of over 2000 hospital encounters provides a comprehensive analysis of the dynamics

169 of Klebsiella infection in colonized patients and identifies patient factors that are present at

170 admission and associated with infection. Overall, a small but significant percentage of colonized

171 patients $(4.3 \%)$ progress to infection. These infections are caused by diverse strains of Klebsiella

172 and are predominantly caused by strains present in a rectal swab (80.2\%) at the time of

173 admission. When infection occurs in colonized patients, it is within days to weeks (median 11.5

174 days) from the first positive rectal swab. The risk of infection is influenced, at least in part, by

175 characteristics of the colonized patients themselves. In particular, the overall burden of

176 comorbidities, pre-existing diagnosis of depression, and a low albumin at baseline are significant

177 and independently associated with infection. Identification of colonized patients at high-risk of

178 Klebsiella infection at the time of admission could be used to direct interventions to prevent

179 these infections in our most vulnerable patients.

181 The dynamics of colonization confirm and expand on previous studies showing a strong link

182 between Klebsiella colonization and infection. Indeed, in a smaller pilot study we demonstrated

183 that intensive care patients were frequently colonized (23\%), colonization was associated with

184 infection (5.2\% vs $1.3 \%$ uncolonized patients; OR 4.01), the colonizing isolates were diverse,

185 and infections were caused often by colonizing strains (13/16 paired isolates matched) (6).

186 Similarly, Gorrie et al found colonization rates of 5.9-19\% among intensive care patients, a

187 strong association between colonization and infection (OR 6.9; 13 infections among 27

188 colonized patients) and high diversity of colonizing and infecting isolates (5). The current study

189 focusing on colonized patients refines the results of these smaller studies, demonstrating a 
190 similar rate of infections (4.3\%), high diversity of infecting strains and a more accurate estimate

191 of concordant infections (80.2\%). It also demonstrates that detectable colonization does not

192 capture all of the infection risk, and that patient variables modify this risk.

193

194 The predominance of concordant infections among colonized patients, and the fact that the

195 infecting strain is often present at admission, indicates that infection prevention for Klebsiella

196 should focus on endogenous, patient risk factors. To that end, our study determined that

197 comorbidities, medication history, and routine laboratory results at admission are informative

198 regarding infection risk, with cases having twice the predicted risk compared to controls $(8.61 \%$

199 vs. 4.35\%). Importantly, the model for concordant infection (Table 4) was more parsimonious

200 than the model for all infections (Table 3). Infection risk increased with the Elixhauser

201 comorbidity score, and depression contributed substantially (OR 2.04, CI 1.2-3.41; $P=0.007$ ).

202 While it is not clear in our study if the association is causal, exposure to antidepressant

203 medications is known to significantly alter the gut microbiome (10). Additionally, prior research

204 has identified depression and antidepressant medication use as risk factors $C$. difficile infection,

205 an infection that is mediated through disruption of the gut microbiome (11). Although

206 antidepressant use was associated with Klebsiella infection on unadjusted analysis, it was

207 confounded by other variables. When we added antidepressant use to the final model, it was not

208 statistically significant $(\mathrm{P}=.66)$, nor was it when we replaced depression with antidepressant use

209 in the model $(\mathrm{P}=0.3)$. It is plausible that the gut microbiome mediates the association between

210 depression and subsequent Klebsiella infection that we observed in colonized patients, but this

211 requires further study. 
213 The association between low albumin and infection (OR 2.0, CI 1.15-3.48, P=0.013) was also

214 previously observed for Klebsiella infections in the ICU including both colonized and non-

215 colonized patients (6). Low albumin can be caused by decreased synthesis due to malabsorption,

216 malnutrition or hepatic dysfunction, or due to losses from ascites, nephropathy, or enteropathy

217 (12). Although the pathophysiologic link between low albumin and Klebsiella infection is

218 unclear, this is an informative and readily widely available predictor, ordered by the physician in

$219>90 \%$ of the cohort.

221 In this multi-year study, the rates of colonization wax and wane by month with the highest rates

222 in the summer. This seasonal pattern was previously demonstrated for Klebsiella bacteremia

223 across 4 continents (13), with both higher temperature and humidity being associated with

224 increased rates. The observed seasonality of Klebsiella colonization may in turn cause a

225 seasonality of infection. Indeed, the proportion of cases by season varied from a low of $8.9 \%$ in

226 Winter to a high of $38.9 \%$ in Summer (Table 2), but tracked closely with the overall proportion

227 of colonized patients by season and the risk of infection in a given colonized patient did not vary

228 by season $(P=0.358)$. The seasonality of Klebsiella bacteremia is not seen for the related

229 pathogens Escherichia coli or Serratia (13). Therefore, prevention efforts that focus on

230 identifying environmental reservoirs of Klebsiella that fluctuate with temperature could have a

231 significant impact on overall rates of infection through reduction of the initial colonization event

232 rate.

234 Our study has several limitations including its retrospective design focused on a convenience

235 sample of ICU/oncology ward patients with post-hoc data extraction. We undertook several 
strategies to mitigate this central limitation. We only included variables that we felt sure were

238 infection (Supplementary Methods), and for variables such as vitals, laboratory results, and

239 medication/device exposures we either excluded them from modeling, captured them only if

240 present prior to swab collection, and/or manually confirmed their baseline status by chart review.

241 We also used matching and multivariable modeling to reduce bias/confounding, and we

242 conducted several sensitivity analyses to confirm our main results. We also could not confirm

243 associations for variables that in prior research were found to be associated with Klebsiella

244 infection. A population-based study of Klebsiella bacteremia found male sex, advanced age,

245 dialysis, solid-organ transplantation, chronic liver disease, and cancer were associated with

246 infection (7). We previously found that advanced age was associated with colonization (8). Like

247 with temperature and seasonality, some infection risks may actually represent colonization risks.

248 Although the comorbidities were not categorized in the same way across studies, none of the

249 other risk factors were significantly associated with infection in colonized patients

250 (Supplementary Results). Finally, technical limitations arising from the sensitivity of our

251 plating method and picking 3 colonies per plate for characterization could have led us to miss

252 some patients who were colonized at baseline and to miss a low abundance, concordant strain in

253 infected patients with detectable colonization.

255 Although the models of Klebsiella infection had significant explanatory power, there are likely to

256 be additional factors that modulate the risk of infection in colonized patients. Relative abundance

257 of Klebsiella colonization is also associated with infection, even after controlling for the

258 variables identified here (co-submitted manuscript), and it could further refine infection risk in 
259 colonized patients. In addition, the complex genomics of Klebsiella is likely to be an important

260 predictor of infection, and we have previously identified several putative pathogenicity loci (9).

261 The present study can further inform such future work by identifying the most important clinical

262 variables that should be considered as confounders when assessing the independent contribution

263 of bacterial genetic loci, colonization density and other features of the gut microbial community

264 on risk of subsequent Klebsiella infection. Such a holistic approach to modeling the risk of

265 infection, from host characteristics to the gut community to the genetics of the colonizing strain,

266 may be what is needed to develop the most accurate and useful models of invasive Klebsiella

267 infection. 


\section{Methods}

270 Cohort identification, sample collection, and strain typing

271 This study was approved by the Institutional Review Boards of the University of Michigan

272 Medical School (IRBMED). We sequentially enrolled subjects to establish a cohort of patients

273 with Klebsiella (K. pneumoniae or K. variicola) rectal colonization. Inpatients admitted to the

274 intensive care units [ICUs] and the oncology wards of the University of Michigan hospitals in

275 Ann Arbor, Michigan, from May 2017 to September 2018, were eligible for inclusion if

276 discarded vancomycin resistant Enterococcus [VRE] screening rectal swab media was available

277 for culture. All patients admitted to these wards undergo screening for rectal VRE carriage per

278 hospital policy, using a flocked swab placed into Amies transport media (E-swab, Becton

279 Dickinson, Franklin Lakes, NJ). Subjects were subsequently enrolled into our study if $K$.

280 pneumoniae was isolated from the swab media through plating on MacConkey agar followed by

281 taxonomic identification using MALDI TOF mass spectrometry. Up to 3 isolates per rectal swab

282 culture were archived along with all clinical culture isolates positive for K. pneumoniae. Wzi

283 typing was done using PCR and Sanger sequencing (14). Wzi type was assigned by uploading

284 the consensus gene sequence to the BigsDB database (https://bigsdb.pasteur.fr).

286 Colonization rates by month

287 The percentage of rectal swabs containing Klebsiella was calculated for each month from records

288 of MacConkey agar growth and MALDI-TOF results for species identification. Average daily

289 temperature for the month was obtained for Ann Arbor from www.wunderground.com.

$291 \quad$ Clinical data extraction and case definitions 
292 Structured queries were used to abstract clinical data from the electronic medical record

293 including demographics, pre-existing comorbidities, vitals, laboratory results, imaging and

294 procedure reports. These clinical data were used to construct variables comprised of baseline

295 features present at or before the time of swab collection. As described further in our results,

296 certain variables were collapsed into colligative variables for the unadjusted and adjusted

297 analyses. Positive clinical cultures for Klebsiella species from the day of, to 90 days after, rectal

298 swab collection were independently reviewed for alignment with our case definitions by two

299 clinician study team members (J.B. and M.B), and disagreements were adjudicated by a third

300 clinician team member (K.R.). All patients with a blood culture growing Klebsiella were

301 considered to have an infection. Manual chart review was conducted by the study team to decide

302 if potential cases met published infection criteria from professional societies / governmental

303 organizations for pneumonia or urinary tract infection (15-19), and cultures from other sites,

304 such as wounds, were considered cases based on the clinical judgment of reviewers. For those

305 meeting clinical case definitions of infection, the clinical isolate and preceding rectal swab

306 isolates were evaluated for concordance of Klebsiella isolates by wzi gene sequencing as

307 previously described $(6,14)$. We have previously demonstrated that wzi sequencing has similar

308 discriminatory power to 7-gene multi-locus sequence typing (6).

310 Statistical Methods

311 Initial descriptive statistics, visualizations such as histograms, and bivariable relationships

312 between predictors and the primary outcome of invasive infection were calculated and used to

313 inform variable construction and inclusion for multivariable modeling. Bivariable relationships

314 were assessed with Student's t-tests (or ANOVA first if multiple categories) and Chi-squared 
315 tests. Given the primary outcome occurring at low frequency and the resulting statistical power

316 considerations, we sought to collapse variables into colligative metrics where applicable. For

317 example, a weighted Elixhauser score (20) was included for modeling in lieu of most individual

318 comorbidities, save when the weight was set to zero or if the weight differed notably in the

319 direction/magnitude from what was observed on bivariable associations with the primary

320 outcome. High-risk antibiotics were defined as third- or fourth-generation cephalosporins,

321 fluoroquinolones, lincosamides, $\beta$-lactam/ $\beta$-lactamase inhibitor combinations, oral vancomycin,

322 and carbapenems, based on their association with disruption of the intestinal microbiome (21).

323 We first constructed an explanatory model to identify independent predictors of the primary

324 outcome. We did this using a modified purposeful selection approach (22) to allow for inclusion

325 of variables in the final model that adjust for confounding in other covariates, even if not

326 independently associated with the primary outcome themselves (see Supplementary Methods).

327 Following this, we constructed additional models to test hypotheses about specific risk factors

328 that were not selected by the above procedure and to conduct sensitivity analyses using the

329 primary model from the above procedure. The sensitivity analyses included limiting the case

330 definition to only apply when isolates from clinical infections were concordant with the

331 colonizing strain by wzi sequencing; and assessing how colonizing species influences risk of

332 infection. All analyses were conducted in R version 4.0.3 (R Foundation for Statistical

333 Computing, Vienna, Austria). A cutoff $P$-value of <.05 was used for statistical inference. Model

334 performance was further assessed using the area under the receiver operator characteristic curve

335 (AuROC) and the discrimination slope using the $\mathrm{R}$ package $p R O C$ (23). 
medRxiv preprint doi: https://doi.org/10.1101/2021.02.23.21251995; this version posted February 27, 2021. The copyright holder for this preprint (which was not certified by peer review) is the author/funder, who has granted medRxiv a license to display the preprint in perpetuity.

It is made available under a CC-BY-NC-ND 4.0 International license .

338 Deidentified data from human subjects may be made available upon request, pending approval

339 from the University of Michigan Institutional Review Board. 
medRxiv preprint doi: https://doi.org/10.1101/2021.02.23.21251995; this version posted February 27, 2021. The copyright holder for this preprint (which was not certified by peer review) is the author/funder, who has granted medRxiv a license to display the preprint in perpetuity.

It is made available under a CC-BY-NC-ND 4.0 International license .

\section{Acknowledgements}

341 Research reported in this publication was supported by the National Institute Of Allergy And

342 Infectious Diseases of the National Institutes of Health under Award Number R01AI125307. The

343 content is solely the responsibility of the authors and does not necessarily represent the official

344 views of the National Institutes of Health. 


\section{References}

346 1. Magill SS, Edwards JR, Bamberg W, Beldavs ZG, Dumyati G, Kainer MA, Lynfield R, 347 Maloney M, McAllister-Hollod L, Nadle J, Ray SM, Thompson DL, Wilson LE, Fridkin 348 SK. 2014. Multistate point-prevalence survey of health care-associated infections. N Engl $349 \quad$ J Med 370:1198-208.

350 2. Wyres KL, Lam MMC, Holt KE. 2020. Population genomics of Klebsiella pneumoniae. $351 \quad$ Nat Rev Microbiol 18:344-359.

352 3. Maatallah M, Vading M, Kabir MH, Bakhrouf A, Kalin M, Naucler P, Brisse S, Giske CG. 2014. Klebsiella variicola is a frequent cause of bloodstream infection in the stockholm area, and associated with higher mortality compared to K. pneumoniae. PLoS

4. Selden R, Lee S, Wang WL, Bennett JV, Eickhoff TC. 1971. Nosocomial Klebsiella infections: intestinal colonization as a reservoir. Ann Intern Med 74:657-64.

358 5. Gorrie CL, Mirceta M, Wick RR, Edwards DJ, Thomson NR, Strugnell RA, Pratt NF, Garlick JS, Watson KM, Pilcher DV, McGloughlin SA, Spelman DW, Jenney AWJ, Holt KE. 2017. Gastrointestinal Carriage Is a Major Reservoir of Klebsiella pneumoniae Infection in Intensive Care Patients. Clin Infect Dis 65:208-215.

362 6. Martin RM, Cao J, Brisse S, Passet V, Wu W, Zhao L, Malani PN, Rao K, Bachman MA. 2016. Molecular Epidemiology of Colonizing and Infecting Isolates of Klebsiella pneumoniae. mSphere 1:e00261-16.

365 7. Meatherall BL, Gregson D, Ross T, Pitout JD, Laupland KB. 2009. Incidence, risk factors, and outcomes of Klebsiella pneumoniae bacteremia. Am J Med 122:866-73. 
367 8. Collingwood A, Blostein F, Seekatz AM, Wobus CE, Woods RJ, Foxman B, Bachman

368 MA. 2020. Epidemiological and Microbiome Associations Between Klebsiella pneumoniae and Vancomycin-Resistant Enterococcus Colonization in Intensive Care Unit Patients. Open Forum Infect Dis 7:ofaa012.

371 9. Martin RM, Cao J, Wu W, Zhao L, Manthei DM, Pirani A, Snitkin E, Malani PN, Rao K, Bachman MA. 2018. Identification of Pathogenicity-Associated Loci in Klebsiella pneumoniae from Hospitalized Patients. mSystems 3:mSystems00015-18.

374 10. Maier L, Pruteanu M, Kuhn M, Zeller G, Telzerow A, Anderson EE, Brochado AR,

377 11. Rogers MA, Greene MT, Young VB, Saint S, Langa KM, Kao JY, Aronoff DM. 2013. Med 11:121.

12. Mc Pherson RA. 2007. Specific Proteins, p 236. In McPherson RA, Pincus MR (ed), Henry’s Clinical Diagnosis and Management by Laboratory Methods. Saunders Elsevier,

383 13. Anderson DJ, Richet H, Chen LF, Spelman DW, Hung YJ, Huang AT, Sexton DJ, Raoult D. 2008. Seasonal variation in Klebsiella pneumoniae bloodstream infection on 4 continents. J Infect Dis 197:752-6. 
15. American Thoracic S, Infectious Diseases Society of A. 2005. Guidelines for the management of adults with hospital-acquired, ventilator-associated, and healthcareassociated pneumonia. Am J Respir Crit Care Med 171:388-416.

392 16. CDC. 2016. Urinary tract infection (catheter-associated urinary tract infection [CAUTI] and non-catheter-associated urinary tract infection [UTI]) and other urinary system

17. Hooton TM, Bradley SF, Cardenas DD, Colgan R, Geerlings SE, Rice JC, Saint S, 2010. Diagnosis, prevention, and treatment of catheter-associated urinary tract infection in adults: 2009 International Clinical Practice Guidelines from the Infectious Diseases Society of America. Clin Infect Dis 50:625-63.

19. Centers for Disease Control and Prevention. . . Centers for Disease Control and America/American Thoracic Society consensus guidelines on the management of the Elixhauser comorbidity measures into a point system for hospital death using administrative data. Med Care 47:626-33. 
412 21. Baggs J, Jernigan JA, Halpin AL, Epstein L, Hatfield KM, McDonald LC. 2018. Risk of

413 Subsequent Sepsis Within 90 Days After a Hospital Stay by Type of Antibiotic Exposure.

$414 \quad$ Clin Infect Dis 66:1004-1012.

415 22. Bursac Z, Gauss CH, Williams DK, Hosmer DW. 2008. Purposeful selection of variables 416 in logistic regression. Source Code Biol Med 3:17.

417 23. Robin X, Turck N, Hainard A, Tiberti N, Lisacek F, Sanchez JC, Muller M. 2011. pROC: an open-source package for $\mathrm{R}$ and $\mathrm{S}+$ to analyze and compare ROC curves. BMC Bioinformatics 12:77. 
Table 1. Summary of Klebsiella infections by species and strain type

\begin{tabular}{|l|l|l|l|l|l|}
\hline & Bacteremia & Pneumonia & Urinary & Other & Total \\
tract & & & \\
infection & & & \\
\hline $\begin{array}{l}\text { Cases of infection (\% of } \\
\text { total) }\end{array}$ & $51(33.6)$ & $40(26.3)$ & $46(30.3)$ & $15(9.9)$ & 152 \\
\hline $\begin{array}{l}\text { Unique cases within 90 days } \\
\text { of enrollment swab (\% of } \\
\text { total) }\end{array}$ & $37(41.1)$ & $27(30.0)$ & $22(24.4)$ & $4(4.4)$ & 90 \\
\hline $\begin{array}{l}\text { Species causing infection } \\
\text { (n,\% of unique cases by site) }\end{array}$ & $33(89.2)$ & $24(88.9)$ & $18(81.8)$ & $3(75.0)$ & $78(86.7)$ \\
\hline $\begin{array}{l}\text { K. pneumoniae } \\
\text { K. variicola }\end{array}$ & $4(10.8)$ & $3(11.1)$ & $4(18.2)$ & $1(25.0)$ & $12(13.3)$ \\
\hline $\begin{array}{l}\text { Total unique wzi types } \\
\text { days between concordant } \\
\text { cases by site) }\end{array}$ & 29 & 25 & 21 & 3 & 68 \\
\hline $\begin{array}{l}\text { Unknown wzi type } \\
\text { infection (n, } \% \text { of unique } \\
\text { swab within } 90 \text { days prior to }\end{array}$ & 1 & 0 & 0 & 1 & 2 \\
\hline Cases with concordant rectal & $30(85.7)$ & $17(65.4 \%)$ & $19(86.3)$ & 3 & $69(80.2)$ \\
\hline
\end{tabular}


medRxiv preprint doi: https://doi.org/10.1101/2021.02.23.21251995; this version posted February 27, 2021. The copyright holder for this preprint (which was not certified by peer review) is the author/funder, who has granted medRxiv a license to display the preprint in perpetuity.

\begin{abstract}
It is made available under a CC-BY-NC-ND 4.0 International license .
\end{abstract}
423

\begin{tabular}{|l|l|l|l|l|l|}
\hline colonization and infection & $67)$ & & $(24,1-$ & $0-7)$ & $0-90)$ \\
& & $90)$ & & \\
\hline
\end{tabular}

424 
Table 2. Demographics and selected baseline ${ }^{1}$ characteristics

\begin{tabular}{|c|c|c|c|}
\hline & $\begin{array}{c}\text { Colonized } \\
(\mathrm{N}=1997)\end{array}$ & $\begin{array}{c}\text { Case } \\
(\mathbf{N}=90)\end{array}$ & $\boldsymbol{P}$ \\
\hline \multicolumn{4}{|l|}{ Age } \\
\hline Mean (SD) & $60.4(15.9)$ & $\begin{array}{c}60.9 \\
(12.9)\end{array}$ & 0.701 \\
\hline Median [Min, Max] & $63.0[1.00,100]$ & $\begin{array}{c}62.0 \\
{[25.0} \\
86.0]\end{array}$ & \\
\hline \multicolumn{4}{|l|}{ Gender } \\
\hline Female & $883(44.2 \%)$ & $\begin{array}{c}41 \\
(45.6 \%)\end{array}$ & 0.802 \\
\hline Male & $1114(55.8 \%)$ & $\begin{array}{c}49 \\
(54.4 \%)\end{array}$ & \\
\hline \multicolumn{4}{|l|}{ Race } \\
\hline Non-white & $335(16.8 \%)$ & $\begin{array}{c}12 \\
(13.3 \%)\end{array}$ & 0.391 \\
\hline White & $1662(83.2 \%)$ & $\begin{array}{c}78 \\
(86.7 \%)\end{array}$ & \\
\hline \multicolumn{4}{|c|}{ Weighted Elixhauser Score } \\
\hline Mean (SD) & $17.1(11.9)$ & $\begin{array}{c}22.6 \\
(11.1)\end{array}$ & $\begin{array}{c}<0.00 \\
1\end{array}$ \\
\hline Median [Min, Max] & $16.0[-14.0,70.0]$ & $\begin{array}{c}22.5 \\
{[1.00,} \\
51.0]\end{array}$ & \\
\hline \multicolumn{4}{|l|}{ Depression } \\
\hline No & $1558(78.0 \%)$ & $\begin{array}{c}59 \\
(65.6 \%)\end{array}$ & 0.006 \\
\hline Yes & $439(22.0 \%)$ & $\begin{array}{c}31 \\
(34.4 \%)\end{array}$ & \\
\hline \multicolumn{4}{|c|}{ Uncomplicated Diabetes } \\
\hline No & $1529(76.6 \%)$ & $\begin{array}{c}62 \\
(68.9 \%)\end{array}$ & 0.094 \\
\hline Yes & $468(23.4 \%)$ & $\begin{array}{c}28 \\
(31.1 \%)\end{array}$ & \\
\hline
\end{tabular}

\section{Complicated Hypertension}


Table 2. Demographics and selected baseline ${ }^{1}$ characteristics

\begin{tabular}{cccc}
\hline & $\begin{array}{c}\text { Colonized } \\
(\mathbf{N = 1 9 9 7 )}\end{array}$ & $\begin{array}{c}\text { Case } \\
(\mathbf{N = 9 0 )}\end{array}$ & $\boldsymbol{P}$ \\
\hline No & $1375(68.9 \%)$ & $\begin{array}{c}54 \\
(60.0 \%)\end{array}$ & 0.077 \\
Yes & & 36 & \\
& $622(31.1 \%)$ & $(40.0 \%)$ &
\end{tabular}

Uncomplicated Hypertension

No

Yes

Urinary catheter at baseline

Yes

No

Feeding tube at baseline

Yes

No

Ventilator at baseline

Yes

No

Central venous catheter at baseline

Yes

$878(44.0 \%)$

No

$1119(56.0 \%)$

Prior diuretic use

No

$1684(84.3 \%)$

Yes

$1684(84.3 \%)$

$313(15.7 \%)$

$\begin{array}{cc}947(47.4 \%) & 52 \\ & (57.8 \%) \\ 1050(52.6 \%) & 38 \\ & (42.2 \%)\end{array}$

$1162(58.2 \%)$ (68.9\%)

(31.1\%)
(58.9\%)

$1924(96.3 \%) \quad(92.2 \%)$
62
0.044
28
0.054
$7(7.8 \%) \quad 0.046$
83
42
0.1
48
37
0.593
53


Table 2. Demographics and selected baseline ${ }^{1}$ characteristics

\begin{tabular}{lccc}
\hline & Colonized & Case & $P$ \\
$(\mathrm{~N}=1997)$ & $(\mathrm{N}=90)$ & $P$ \\
\hline
\end{tabular}

\section{Prior PPI use}

No

Yes

Prior vitamin D use

No

Yes

Prior use of pressors/inotropes

No

Yes

Prior use of antidepressants/antipsychotics

No

Yes

Prior use of histamine antagonists

No

Yes

Prior high risk antibiotic use ${ }^{2}$

No

Yes

Baseline circulating WBC (thousands of cells/microliter)

Mean (SD)

$$
1637(82.0 \%)
$$

$360(18.0 \%)$

58
$(64.4 \%)$
32
$(35.6 \%)$

69

$<0.00$

21

$1854(92.8 \%)$

$143(7.2 \%)$

66

$<0.00$

24

(26.7\%)

1

1
$<0.00$
1

66

(73.3\%)

$<0.00$

24

(26.7\%)

$259(13.0 \%)$

68

$<0.00$

(75.6\%)

1

22

(24.4\%)

$244(12.2 \%)$

(24.4\%)

56

(62.2\%)

$<0.00$

$1699(85.1 \%)$

34

$298(14.9 \%)$

(37.8\%) 
Table 2. Demographics and selected baseline ${ }^{1}$ characteristics

\begin{tabular}{|c|c|c|c|}
\hline & $\begin{array}{l}\text { Colonized } \\
(\mathrm{N}=1997)\end{array}$ & $\begin{array}{c}\text { Case } \\
(\mathrm{N}=90)\end{array}$ & $\boldsymbol{P}$ \\
\hline Median [Min, Max] & $6.00[0.0900,160]$ & $\begin{array}{c}5.50 \\
{[0.0900} \\
15.5]\end{array}$ & \\
\hline Missing & $14(0.7 \%)$ & $0(0 \%)$ & \\
\hline \multicolumn{4}{|c|}{ Baseline serum hemoglobin (g/dL) } \\
\hline Mean (SD) & $8.74(2.30)$ & $\begin{array}{c}7.58 \\
(1.79)\end{array}$ & $\begin{array}{c}<0.00 \\
1\end{array}$ \\
\hline Median [Min, Max] & $8.30[3.60,17.4]$ & $\begin{array}{c}7.00 \\
{[4.70,} \\
13.4]\end{array}$ & \\
\hline Missing & $14(0.7 \%)$ & $0(0 \%)$ & \\
\hline \multicolumn{4}{|c|}{$\begin{array}{l}\text { Baseline circulating platelets (thousands of } \\
\text { cells/microliter) }\end{array}$} \\
\hline Mean (SD) & $145(97.9)$ & $\begin{array}{c}122 \\
(94.0)\end{array}$ & 0.023 \\
\hline Median [Min, Max] & $134[0,627]$ & $\begin{array}{l}107[1.00, \\
380]\end{array}$ & \\
\hline Missing & $14(0.7 \%)$ & $0(0 \%)$ & \\
\hline \multicolumn{4}{|c|}{ Baseline serum creatinine (mg/dL) } \\
\hline Mean (SD) & $0.870(0.781)$ & $\begin{array}{c}0.772 \\
(0.495)\end{array}$ & 0.078 \\
\hline Median [Min, Max] & $0.700[0.0900,15.8]$ & $\begin{array}{c}0.660 \\
{[0.160} \\
3.61]\end{array}$ & \\
\hline Missing & $19(1.0 \%)$ & $0(0 \%)$ & \\
\hline
\end{tabular}

\section{Serum albumin}

$\begin{array}{lccc}<2.5 \mathrm{~g} / \mathrm{dL} & 353(17.7 \%) & 39 & <0.00 \\ & & (43.3 \%) & 1 \\ >=2.5 \mathrm{~g} / \mathrm{dL} & 1456(72.9 \%) & (52.2 \%) \\ \text { Missing } & 188(9.4 \%) & 4(4.4 \%)\end{array}$

\section{Site of clinical infection}

other

$0(0 \%) \quad 4(4.4 \%)$ 


\section{Table 2. Demographics and selected baseline ${ }^{1}$ characteristics}

\begin{tabular}{|c|c|c|c|}
\hline & $\begin{array}{c}\text { Colonized } \\
(\mathrm{N}=1997)\end{array}$ & $\begin{array}{c}\text { Case } \\
(\mathrm{N}=90)\end{array}$ & $\boldsymbol{P}$ \\
\hline blood & $0(0 \%)$ & $\begin{array}{c}37 \\
(41.1 \%)\end{array}$ & - \\
\hline respiratory & $0(0 \%)$ & $\begin{array}{c}27 \\
(30.0 \%)\end{array}$ & - \\
\hline Urine & $0(0 \%)$ & $\begin{array}{c}22 \\
(24.4 \%)\end{array}$ & - \\
\hline none & 1997 & & - \\
\hline Season of baseline rectal swab colonization & & & 0.38 \\
\hline Winter (January-March) & $248(12.4 \%)$ & $\begin{array}{l}8(8.9 \% \\
\text { of cases, } \\
3.1 \% \text { of } \\
\text { Winter } \\
\text { swabs })\end{array}$ & \\
\hline Spring (April-June) & $503(25.2 \%)$ & $\begin{array}{c}28 \\
(31.1 \% \text { of } \\
\text { cases, } \\
5.3 \% \text { of } \\
\text { Spring } \\
\text { swabs) }\end{array}$ & \\
\hline Summer (July-September) & $717(35.9 \%)$ & $\begin{array}{c}35 \\
\text { (38.9\% of } \\
\text { cases, } \\
4.7 \% \text { of } \\
\text { Summer } \\
\text { swabs) }\end{array}$ & \\
\hline Fall (October-December) & $529(26.5 \%)$ & $\begin{array}{c}19 \\
(21.1 \% \text { of } \\
\text { cases, } \\
3.5 \% \text { of } \\
\text { Fall } \\
\text { swabs) }\end{array}$ & \\
\hline \multicolumn{4}{|c|}{$\begin{array}{l}{ }^{1} \text { Features considered to be baseline if present }>48 \text { hours but }<90 \text { days prior to rectal swab } \\
\text { collection. } \\
{ }^{2} \text { High-risk antibiotics were defined as third- or fourth-generation cephalosporins, } \\
\text { fluoroquinolones, lincosamides, } \beta \text {-lactam/ } \beta \text {-lactamase inhibitor combinations, oral } \\
\text { vancomycin, and carbapenems. }\end{array}$} \\
\hline
\end{tabular}


Table 3. Model of Klebsiella infection in colonized patients.

\section{Variable}

Elixhauser Score (weighted)

Depression

Prior $^{1}$ diuretic use

Prior $^{1}$ vitamin D use

Prior $^{1}$ use of pressors/inotropes

Prior $^{1}$ use of high-risk antibiotics

Albumin $<2.5 \mathrm{~g} / \mathrm{dL}$

${ }^{1}$ Features considered to be baseline if present $>48$ hours but $<90$ days prior to rectal swab collection.

\section{OR $[95 \% \mathrm{CI}]$}

$1.02[1.00,1.04]$

$1.80[1.12,2.88]$

$1.28[0.70,2.29]$

$1.36[0.71,2.57]$

$1.9[0.96,3.71]$

$1.38[0.74,2.52]$

.298

$2.13[1.3,3.48]$

.003 
Table 4. Model for concordant Klebsiella infections (retaining cases without concordance)

Variable

Weighted Elixhauser

Depression

Baseline ${ }^{1}$ Hemoglobin

Albumin $<2.5 \mathrm{~g} / \mathrm{dL}$

${ }^{1}$ Features c
collection.
OR [95\% CI $]$

$1.03[1.01,1.05]$

$2.04[1.2,3.41]$

$0.88[0.75,1.01]$

$2[1.15,3.48]$ 


\section{$434 \quad$ Figures}

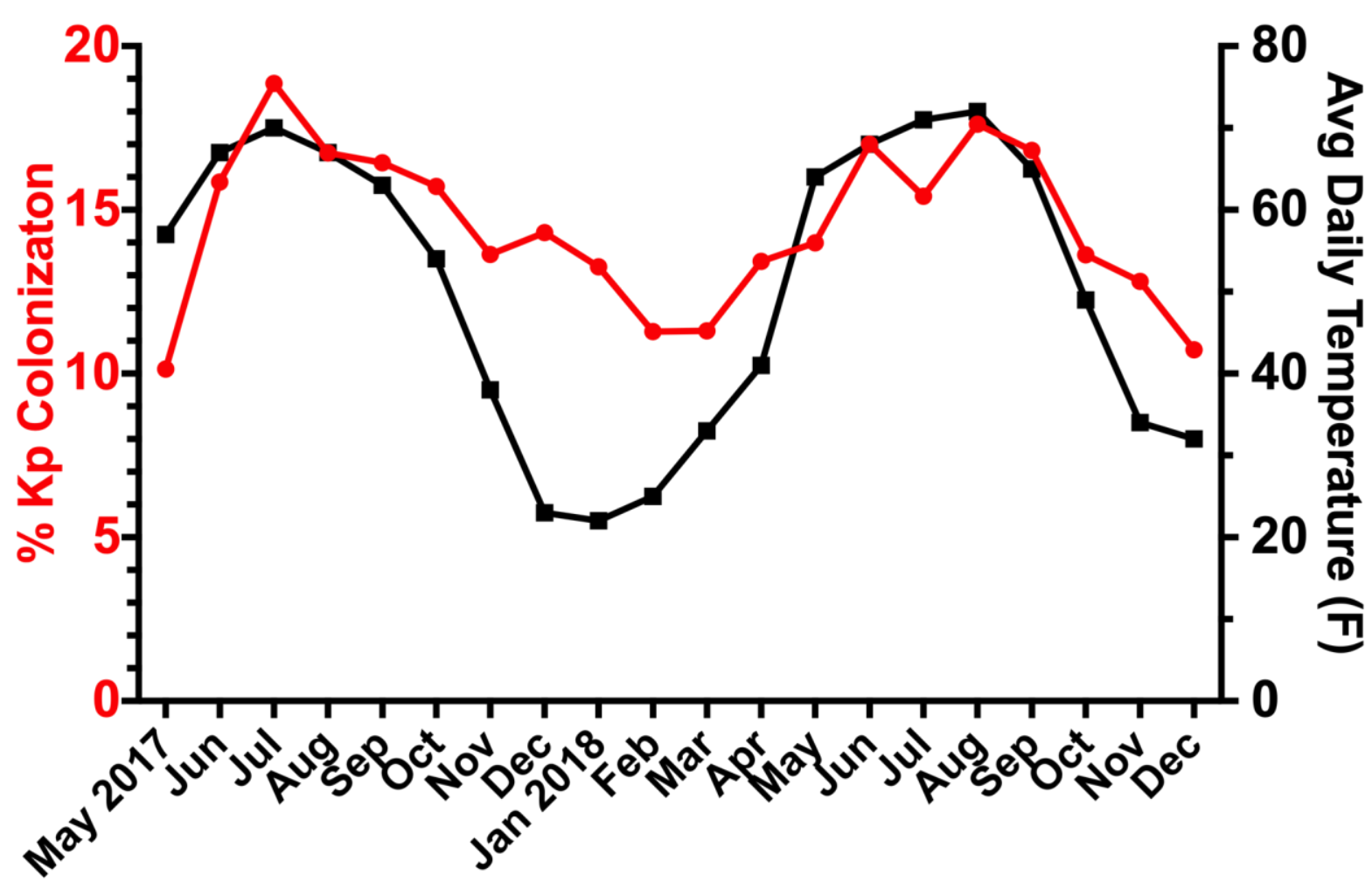
with the average daily temperature for that month. 
medRxiv preprint doi: https://doi.org/10.1101/2021.02.23.21251995; this version posted February 27, 2021. The copyright holder for this preprint (which was not certified by peer review) is the author/funder, who has granted medRxiv a license to display the preprint in perpetuity.

It is made available under a CC-BY-NC-ND 4.0 International license.

444 Figure 2. Boxplots of time to concordant infection vs. site of infection. Given the significant

445 right skew of the data and for better visibility, the number of days is plotted on a log scale. The

446 boxes show the medians and interquartile ranges (IQRs), while the whiskers show 1.5x the IQRs.

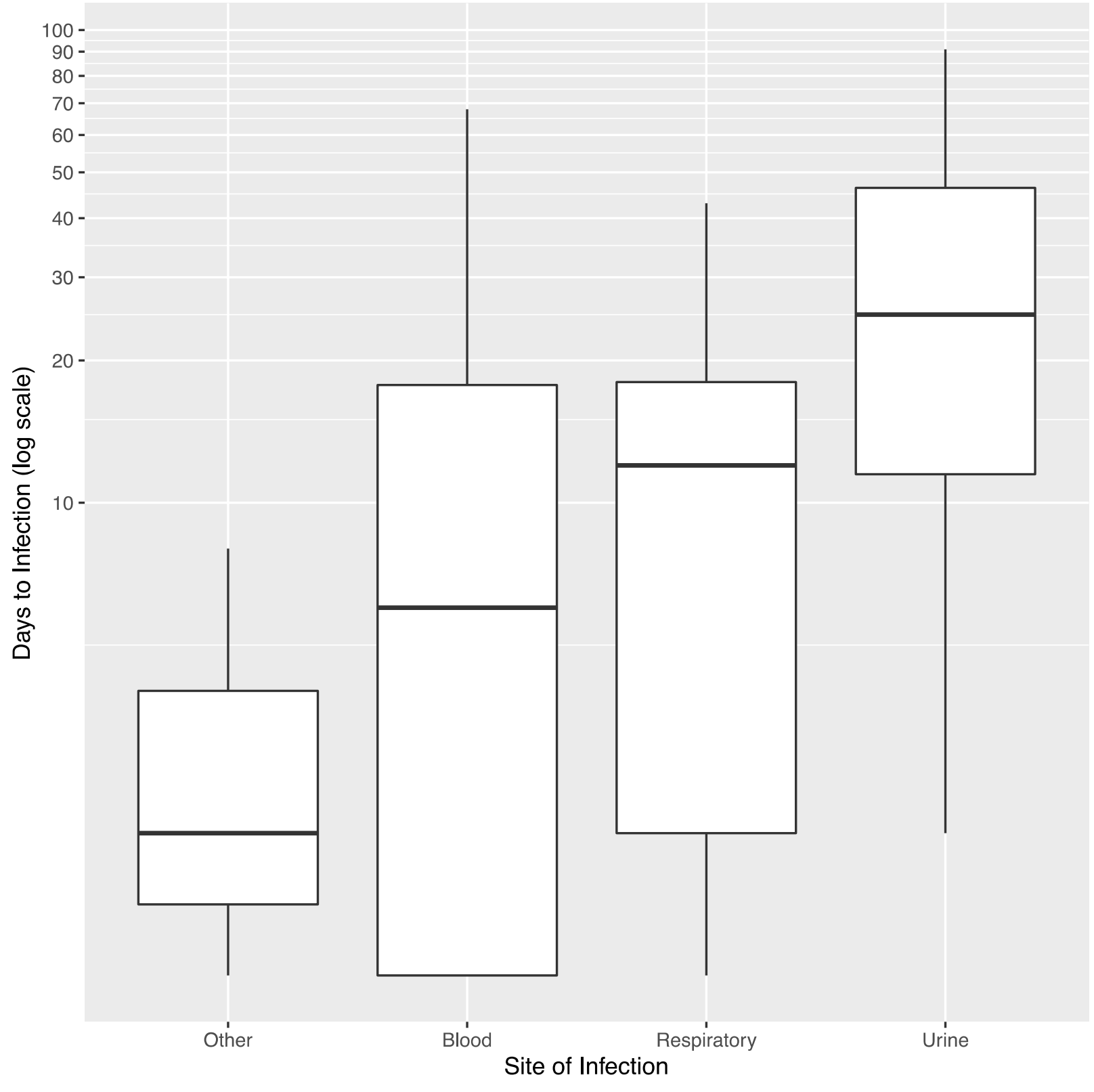

